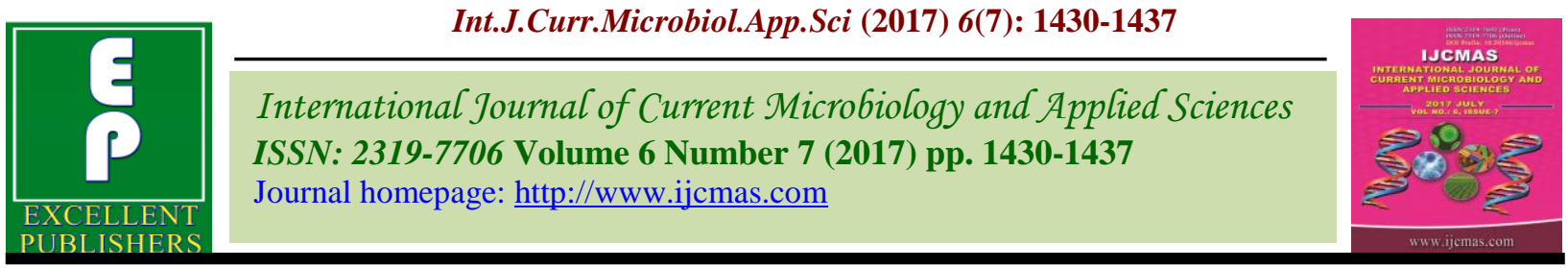

Original Research Article

https://doi.org/10.20546/ijcmas.2017.607.171

\title{
Line $x$ Tester Yanalysis in Maize (Zea mays L.) for Various Morpho-Agronomic Traits under Temperate Conditions
}

\author{
Z.A. Dar", A.A. Lone, N.S. Khuroo, G. Ali, I. Abidi, M.A. Ahangar, \\ M.A. Wani, A.B. Yasin, A. Gazal, R.A. Lone, N. Yousuf and S. Gulzar \\ DARS, Budgam (SKUAST-K) Srinagar, India \\ *Corresponding author
}

\section{A B S T R A C T}

The present study was aimed to assess the general combining ability of parents

Keywords

Combining ability, gca, sca, Line $x$ tester, Maize, Yield.

Article Info

Accepted:

17 June 2017

Available Online:

10 July 2017 and specific combining ability of their crosses for yield and yield related traits. Eight inbred lines were crossed with three testers using line $\times$ tester mating design. The resulting twenty-four crosses along with eleven parents were evaluated at two locations during Kharif 2015 in a complete randomized block design. The parent KDM-445A was identified as best combiner for grain yield plant ${ }^{-1}$ followed by CM-502 and KDM-347. Besides, KDM-445A was also accompanied with significant and desirable gca for 100 grain weight, grain depth, shelling percentage, protein content, number of kernels row $^{-1}$, days to 50 per cent tasseling and days to 50 per cent silking. Among the crosses, KDM-347 $\times$ SMC-7, KDM$445 \mathrm{~A} \times$ DMR-N6 and V-351 $\times$ DMR-N6 exhibited highly significant and desirable SCA effects for grain yield plant ${ }^{-1}$. Therefore, these crosses can be utilized for developing high yielding hybrid varieties in maize.

\section{Introduction}

Maize is considered one of the major cereals in the world with an annual production of about 854.6 million tonnes over an area of 168.4 million hectares and average productivity of 5.07 tonnes per hectare (Anonymous, 2015). It has assumed greater significance due to its demand for food, feed and industrial utilization. It is a versatile crop with wider genetic variability and able to grow successfully in wider range of environments. Hybrid vigour has been exploited to its fullest in maize crop. However, not all the parental lines upon hybridization exhibit economically exploitable heterosis in their hybrids. This emphasizes the importance of combining ability analysis. The concept of combining ability analysis plays a significant role in crop improvement as it helps in characterizing the nature and magnitude of genetic effects governing yield and its component traits besides pin pointing the promising parents to be used in the synthesis of superior hybrids and populations, particularly when the production of hybrids is not feasible due to some inherent problems in economic hybrid seed production. In biometrical genetics two types combining abilities are considered i.e., general combining ability (GCA) and specific combining ability (SCA). General combining ability is the average performance of a strain in series of cross combinations estimated 
from the performance of $F_{1}$ 's from the crosses, whereas specific combining ability is used to designate those cases in which certain combinations do relatively better or worse than would be expected on the basis of average performance of lines involved. Line $x$ tester mating design was developed by Kempthorne (1957), which provides valuable information on general and specific combining ability effects of parents and their hybrid combination. In maize, combining ability analysis was carried out by several workers (Iqbal et al., 2007; Shashidhara, 2008; Kanagarasu et al., 2010; Shanthi et al., 2010; Premlatha et al., 2011; Abuali et al., 2012; Haddabi et al., 2013 and Krupakar et al., 2014). The present study was therefore, undertaken with a view to estimate general combining ability of parents and specific combining ability of their crosses for yield and its component traits in maize.

\section{Materials and Methods}

The basic material for the present investigation comprised of eight diverse maize inbred lines viz., V-351, V-335, V-370, CM-502, KDM-500, KDM-347, KDM-440B and KDM-445A and three testers viz., SMC7, CML-451, and DMR-N6. Twenty four crosses were obtained from the set of parents using the methodology of line $\times$ tester mating design.as suggested by Kempthorne (1957) during rabi 2014-15 at Winter Nursery Centre, Hyderabad. Twenty four crosses along with their parents were evaluated at two locations viz. Main campus, SKUAST-K and Dryland Agriculture Research Station, Budgam during Kharif 2015 in a complete randomized block design with two replications at both the locations. The experimental plot comprised of two rows each of 4 metre length with a planting geometry of $75 \times 20 \mathrm{~cm}$. All the Recommended agronomic practices were followed to raise a good crop. Observations on various traits viz., plant height $(\mathrm{cm})$, ear height $(\mathrm{cm})$, grain depth, number of kernel rows ear ${ }^{-1}$, number of kernels row $^{-1}$, shelling percentage $(\%), 100$ grain weight $(\mathrm{g})$, prolificacy index, grain yield plant $^{-1}(\mathrm{~g})$ and protein content (\%) were recorded on five randomly selected competitive plants except for maturity traits (days to 50 per cent tasseling and days to 50 per cent silking) where data were recorded on plot basis.

Data from each location was subjected to ANOVA separately to detect the significance of genotypic differences before a combined ANOVA. Combining ability analysis (pooled over environments) was carried out according to model as suggested by Kempthorne (1957). Data was analysed using Windostat (9.1) software.

\section{Results and Discussion}

Analysis of variance for the traits under study in pooled analysis is presented in table 1. Perusal of the results revealed significant differences among parents (lines), crosses and line $\times$ testers for all traits under study, indicating that the material selected was diverse for all the traits under study. Significant LxT component suggested presence of high SCA among hybrids. It was also found that mean squares due to lines were larger when compared to testers for all the traits indicating greater diversity among lines. This was in confirmation with the results reported by Abuali et al., (2012). Analysis of variance for combining ability revealed significant mean squares for GCA and SCA for all the traits studied which indicated importance of both additive and non-additive gene action in the inheritance of these traits. The knowledge of combining ability effects of parents and crosses together with per se performance is of paramount importance to a breeder for isolating desirable lines and selection of an appropriate 
methodology for handling the segregating generations. The general and specific combining ability of the parents and crosses respectively were estimated and discussed below:

\section{General combining ability effects}

The GCA effects represent the additive nature of the gene action. In the present study, the general combining ability of parents (8 lines) were estimated to know their genetic worth for use in production of superior progenies. The estimates of GCA effects are listed in table 2 and close examination of the results revealed that none of the parents showed significant GCA effects in the desired direction simultaneously for all the traits studied. For grain yield plant ${ }^{-1} \mathrm{KDM}-445 \mathrm{~A}$ was identified as best combiner followed by CM-502 and KDM-347. These can be used directly as parents for developing high yielding single cross hybrids. KDM-445A was also accompanied with significant gca in desired direction for 100 grain weight, grain depth, shelling percentage, protein content kernels row $^{-1}$, flowering traits viz., days to 50 per cent tasseling, days to 50 per cent silking. Similar results significant were reported for yield and yield related traits by Pavan et al., 2011 and Singh et al., 2012.

For flowering traits, V-335, KDM-445A, and KDM-347 were having highly significant negative GCA effects. Desai and Singh (2001) also reported the importance of negative general combining ability estimates for days to 50 per cent tasseling and days to 50 per cent silking for characterising the parents as good combiners. For morphological traits like plant height and ear height V-351, V-335 and KDM-500 showed the desirable GCA effects. For kernel rows $\mathrm{cob}^{-1}, \mathrm{CM}-502$ was found as best combiner which also depicted highest and desirable combining ability for grain yield plant $^{-1}$, kernels row $^{-1}, 100$ grain weight and shelling percentage. High per se performance for kernels row $^{-1}$, an important yield contributing trait was` exhibited by KDM-445A followed by KDM-347 and CM-502. Significant and desirable GCA effects for grain depth were exhibited by CM-502, KDM-500, KDM440B and KDM-445A. For 100 grain weight, CM-502 was found to be best combiner displayed by highly significant positive GCA effects followed by KDM-347 and KDM-500.

Table.1 Analysis of variance for different traits in maize (Zea mays $L$.)

\begin{tabular}{|l|c|c|c|c|c|c|c|}
\hline $\begin{array}{c}\text { Source of } \\
\text { variation }\end{array}$ & d.f. & $\begin{array}{c}\text { Days to } \\
\mathbf{5 0 \%} \\
\text { tasselling }\end{array}$ & $\begin{array}{c}\text { Days to } \\
\mathbf{5 0 \%} \\
\text { silking }\end{array}$ & $\begin{array}{c}\text { Plant height } \\
(\mathbf{c m})\end{array}$ & $\begin{array}{c}\text { Ear height } \\
(\mathbf{c m})\end{array}$ & Prolificacy & $\begin{array}{c}\text { Kernel } \\
\text { rows cob }\end{array}$ \\
\hline Environments & 1 & $1327.593^{* *}$ & $1335.041^{* *}$ & $50592.360^{* *}$ & $18869.237^{* *}$ & 0.001 & $1.181^{* *}$ \\
\hline Replications & 1 & 0.010 & 0.375 & 17.543 & 8.230 & 0.002 & 0.077 \\
\hline Rep $\times$ env & 1 & 1.260 & 1.500 & 0.339 & 4.725 & 0.005 & 0.003 \\
\hline Crosses & 23 & $30.640^{* *}$ & $28.521^{* *}$ & $1368.560^{* *}$ & $735.382^{* *}$ & $0.037^{* *}$ & $6.911^{* *}$ \\
\hline Lines & 7 & $91.166^{*}$ & $98.375^{* *}$ & $2702.331^{*}$ & $1338.545^{*}$ & $0.086^{* *}$ & $23.501^{*}$ \\
\hline Testers & 2 & 31.343 & 28.690 & 618.567 & 1305.800 & 0.030 & 0.203 \\
\hline Line $\times$ tester & 14 & $21.642^{* *}$ & $18.458^{*}$ & $808.817^{* *}$ & $352.312^{* *}$ & $0.013^{* *}$ & $8.116^{* *}$ \\
\hline Crosses $\times$ env & 23 & 0.550 & 0.563 & $26.901^{* *}$ & $32.055^{* *}$ & 0.001 & 0.032 \\
\hline Lines $\times$ env & 7 & 0.712 & $1.113^{*}$ & 42.697 & 26.094 & 0.001 & 0.037 \\
\hline Testers $\times$ env & 2 & 0.875 & 0.541 & 2.198 & 0.173 & 0.002 & 0.040 \\
\hline L $\times$ T $\times$ env & 14 & $0.874 *$ & $1.2300^{*}$ & $22.532^{* *}$ & $39.589^{* *}$ & 0.004 & $0.112^{* *}$ \\
\hline Error & 46 & 0.396 & 0.524 & 4.620 & 2.061 & 0.003 & 0.034 \\
\hline
\end{tabular}




\begin{tabular}{|c|c|c|c|c|c|c|c|}
\hline $\begin{array}{l}\text { Source of } \\
\text { variation }\end{array}$ & d.f. & $\begin{array}{c}\text { Kernels } \\
\text { row }^{-1}\end{array}$ & $\begin{array}{l}\text { Grain } \\
\text { depth } \\
\text { (cm) }\end{array}$ & $\begin{array}{l}100 \text { grain } \\
\text { weight } \\
\text { (g) }\end{array}$ & $\begin{array}{c}\text { Shelling } \\
\text { percentage } \\
(\%)\end{array}$ & $\begin{array}{c}\text { Grain yield } \\
\text { plant }^{-1} \\
(\mathrm{~g})\end{array}$ & $\begin{array}{c}\text { Protein } \\
\text { content } \\
(\%)\end{array}$ \\
\hline Environments & 1 & $149.675^{* *}$ & $0.002 * *$ & $9.519 * *$ & $1.680^{*}$ & $309.350 * *$ & $0.181^{* *}$ \\
\hline Replications & 1 & 2.100 & 0.002 & 0.968 & 0.860 & 26.001 & 0.021 \\
\hline Rep $\times$ env & 1 & 0.974 & 0.004 & 0.457 & 0.255 & 3.884 & 0.0003 \\
\hline Crosses & 23 & $92.341 * *$ & $0.035 * *$ & $43.047 * *$ & $56.440 * *$ & $4083.823 * *$ & $0.789 * *$ \\
\hline Lines & 7 & $190.000^{*}$ & $0.064^{*}$ & $164.001 * *$ & $109.000^{*}$ & $9102.700 *$ & $1.966^{*}$ \\
\hline Testers & 2 & $23.20 * *$ & $0.044 * *$ & 24.679 & 93.014 & 181.286 & 0.262 \\
\hline Line $\times$ tester & 14 & $65.000^{* *}$ & $0.021 * *$ & $38.776^{* *}$ & $36.782 * *$ & $3169.133 * *$ & $0.670^{* *}$ \\
\hline Crosses $\times$ env & 23 & $1.059 * *$ & 0.001 & $0.646^{* *}$ & 0.332 & $27.945^{* *}$ & 0.043 \\
\hline Lines $\times$ env & 7 & 1.426 & 0.001 & 0.736 & 0.428 & $77.530 * *$ & 0.052 \\
\hline Testers $\times$ env & 2 & 0.009 & 0.001 & 0.388 & 0.109 & 12.649 & 0.003 \\
\hline $\mathrm{L} \times \mathrm{T} \times \mathrm{env}$ & 14 & $1.026 * *$ & $0.006 * *$ & $0.638^{* *}$ & 0.315 & $19.675^{* *}$ & $0.044^{*}$ \\
\hline Error & 46 & 0.272 & 0.001 & 0.248 & 0.244 & 6.666 & 0.017 \\
\hline
\end{tabular}

Table.2 General combining ability effects of lines and testers for Different characters in maize (Zea mays L.)

\begin{tabular}{|c|c|c|c|c|c|c|}
\hline Parents & $\begin{array}{c}\text { Days to } \\
\mathbf{5 0 \%} \\
\text { tasselling } \\
\end{array}$ & $\begin{array}{c}\text { Days to } \\
50 \% \\
\text { silking } \\
\end{array}$ & $\begin{array}{c}\text { Plant } \\
\text { height } \\
(\mathrm{cm}) \\
\end{array}$ & $\begin{array}{l}\text { Ear height } \\
\quad(\mathrm{cm})\end{array}$ & Prolificacy & $\begin{array}{c}\text { Kernel } \\
\text { rows cob }^{-1}\end{array}$ \\
\hline \multicolumn{7}{|l|}{ Lines } \\
\hline V-351 & -0.260 & $0.667 * *$ & $-17.967 * *$ & $-15.595 * *$ & $0.195 * *$ & $0.359 * *$ \\
\hline V-335 & $-1.344 * *$ & $-1.500 * *$ & $-10.800 * *$ & $-5.903 * *$ & $-0.055 * *$ & $-0.191 * *$ \\
\hline V-370 & $3.323^{* *}$ & $3.333 * *$ & $10.416^{* *}$ & $7.389 * *$ & 0.020 & $-0.741 * *$ \\
\hline CM-502 & $0.740 * *$ & 0.167 & $2.075 * *$ & $1.930 * *$ & $-0.055 * *$ & $0.951 * *$ \\
\hline KDM-500 & $0.490 * *$ & $0.833 * *$ & $-21.472 * *$ & $-12.511 * *$ & $-0.055 * *$ & $0.492 * *$ \\
\hline KDM-347 & $-1.283 * *$ & $-0.917 * *$ & $5.075 * *$ & $2.689 * *$ & $-0.055 * *$ & $-1.266 * *$ \\
\hline KDM-440B & 0.073 & -0.083 & $17.663 * *$ & $15.397 * *$ & 0.011 & $0.517 * *$ \\
\hline KDM-445A & $-1.427 * *$ & $-1.167 * *$ & $15.011 * *$ & $6.605 * *$ & -0.005 & $-0.121 *$ \\
\hline S.E.g (lines) & 0.182 & 0.209 & 0.620 & -0.414 & 0.016 & 0.053 \\
\hline
\end{tabular}

\begin{tabular}{|l|c|c|c|c|c|c|}
\hline \multicolumn{7}{|l|}{ Testers } \\
\hline SMC-7 & $1.667 * *$ & $1.688^{* * *}$ & $4.725^{* *}$ & $3.730^{* *}$ & $-0.021 *$ & $-0.072^{*}$ \\
\hline CML-451 & $-1.708^{* *}$ & $-1.813 * * *$ & $-3.970^{* *}$ & $-7.376^{* *}$ & -0.015 & -0.013 \\
\hline DMR-N6 & 0.042 & 0.125 & $-0.756^{* *}$ & $3.646^{* *}$ & $0.035^{* *}$ & $0.085^{*}$ \\
\hline S.E.gi(testers) & 0.113 & 0.128 & 0.380 & 0.254 & 0.010 & 0.033 \\
\hline $\begin{array}{l}\text { High gca } \\
\text { parents }\end{array}$ & 4 & 5 & 4 & 4 & 2 & 5 \\
\hline
\end{tabular}

*, ** Significant at 5 and 1 percent levels, respectively 


\begin{tabular}{|l|c|c|c|c|c|c|}
\hline Parents & Kernels row & $\begin{array}{c}\text { Grain depth } \\
(\mathbf{c m})\end{array}$ & $\begin{array}{c}\text { 100 grain } \\
\text { weight }(\mathbf{g})\end{array}$ & $\begin{array}{c}\text { Shelling } \\
\text { percentage } \\
(\mathbf{\%})\end{array}$ & $\begin{array}{c}\text { Grain yield } \\
\text { plant } \\
(\mathbf{g})\end{array}$ & $\begin{array}{c}\text { Protein } \\
\text { content } \\
(\boldsymbol{\%})\end{array}$ \\
\hline Lines & \multicolumn{7}{|l|}{} \\
\hline V-351 & $-0.335^{*}$ & $-0.081^{* *}$ & $-1.062^{* *}$ & $-4.290^{* *}$ & $-6.079^{* *}$ & $0.229^{* *}$ \\
\hline V-335 & $-4.143^{* *}$ & $-0.062^{* *}$ & $-3.337^{* *}$ & $-2.128^{* *}$ & $-30.814^{* *}$ & 0.040 \\
\hline V-370 & -0.205 & $-0.037^{* *}$ & $-2.672^{* *}$ & -0.243 & $-14.469^{* *}$ & $-0.235^{* *}$ \\
\hline CM-502 & $1.123^{* *}$ & $0.057^{* *}$ & $2.571^{* *}$ & $2.451^{* *}$ & $18.253^{* *}$ & $-0268^{* *}$ \\
\hline KDM-500 & $-1.910^{* *}$ & $0.037^{* *}$ & $1.146^{* *}$ & $-1.827^{* *}$ & $2.613^{* *}$ & $-0.518^{* *}$ \\
\hline KDM-347 & $1.312^{* *}$ & $-0.046^{* *}$ & $1.504^{* *}$ & $3.632^{* *}$ & $12.118^{* *}$ & 0.073 \\
\hline KDM-440B & $-1.773^{* *}$ & $0.093^{* *}$ & -0.037 & $2.022^{* *}$ & $-14.066^{* *}$ & $0.357^{* *}$ \\
\hline KDM-445A & $5.931^{* *}$ & $0.046^{* *}$ & $1.888^{* *}$ & $0.383^{*}$ & $32.444^{* *}$ & $0.323^{* *}$ \\
\hline S.E.g.(lines) & 0.151 & $-0.081^{* *}$ & 0.144 & 0.143 & 0.745 & 0.038 \\
\hline
\end{tabular}

\begin{tabular}{|l|c|c|c|c|c|c|}
\hline \multicolumn{7}{|l|}{ Testers } \\
\hline SMC-7 & -0.038 & $-0.044^{* *}$ & $0.605^{* *}$ & $-1.351^{* *}$ & -0.340 & $-0.076^{* *}$ \\
\hline CML-451 & $-0.832^{* *}$ & $0.039^{* *}$ & $0.402^{* *}$ & $1.916^{* *}$ & $-2.192^{* *}$ & $0.100^{* *}$ \\
\hline DMR-N6 & $0.870^{* *}$ & 0.003 & $-1.007^{* *}$ & $-0.565^{* *}$ & $2.532^{* *}$ & -0.025 \\
\hline S.E.gi(testers) & 0.092 & 0.001 & 0.088 & 0.087 & 0.456 & 0.023 \\
\hline $\begin{array}{l}\text { High gca } \\
\text { parents }\end{array}$ & $\mathbf{4}$ & $\mathbf{5}$ & $\mathbf{6}$ & $\mathbf{5}$ & $\mathbf{5}$ & $\mathbf{4}$ \\
\hline
\end{tabular}

*, ** Significant at 5 and 1 percent levels, respectively

Table.3 Specific combining ability effects of lines and testers for Different characters in maize (Zea mays L.)

\begin{tabular}{|c|c|c|c|c|c|c|}
\hline Crosses & $\begin{array}{l}\text { Days to } 50 \% \\
\text { tasselling }\end{array}$ & $\begin{array}{l}\text { Days to } 50 \% \\
\text { silking }\end{array}$ & $\begin{array}{l}\text { Plant height } \\
\text { (cm) }\end{array}$ & $\begin{array}{c}\text { Ear height } \\
\text { (cm) }\end{array}$ & Prolificacy & $\begin{array}{c}\text { Kernel }^{-1} \\
\text { rows cob }^{-1}\end{array}$ \\
\hline $\mathrm{V}-351 \times \mathrm{SMC}-7$ & $2.167 * *$ & $1.729 * *$ & $-22.867 * *$ & $-17.155^{* *}$ & 0.046 & $0.697 * *$ \\
\hline V-351×CML-451 & 0.042 & $0.979 * *$ & $10.353 * *$ & $4.626^{* *}$ & 0.040 & $-1.687 * *$ \\
\hline V-351×DMR-N6 & $-2.208 * *$ & $-2.708 * *$ & $12.514 * *$ & $12.529 * *$ & $-0.085 * *$ & $0.990 * *$ \\
\hline V-335 $\times$ SMC-7 & $-1.500 * *$ & $-1.188 * *$ & $11.466^{* *}$ & $5.153^{* * *}$ & 0.021 & $2.073 * *$ \\
\hline V-335×CML-451 & 0.625 & -0.188 & $12.887 * *$ & $10.109 * *$ & 0.015 & $-1.287 * *$ \\
\hline V-335×KDM343A & $0.875 * *$ & $1.375^{* *}$ & $-24.353 * *$ & $-15.263 * *$ & -0.035 & $-0.785 * *$ \\
\hline V-370 $\times$ SMC-7 & $-1.417 * * *$ & $-1.771 * *$ & $7.775^{* *}$ & $12.986^{* *}$ & -0.054 & -0.102 \\
\hline KDM2111×CML-451 & $-2.042 * *$ & $-0.771^{*}$ & $-6.530 * *$ & $-7.082 * *$ & -0.010 & $1.113^{* *}$ \\
\hline V-370×DMR-N6 & $3.458^{* *}$ & $2.542 * *$ & -1.244 & $-5.904 * *$ & $0.065 *$ & $-1.010^{* *}$ \\
\hline CM-502×SMC-7 & $3.167 * *$ & $2.646^{* *}$ & $6.291 * *$ & 0.570 & 0.021 & $0.281 * *$ \\
\hline KDM2113× CML-451 & $-1.708 * *$ & $-2.104 * *$ & $-4.963 * *$ & $-3.274 * *$ & 0.015 & $1.246^{* *}$ \\
\hline CM-502×DMR-N6 & $-1.458 * *$ & -0.542 & -1.328 & $2.704 * *$ & -0.035 & $-1.527 * *$ \\
\hline
\end{tabular}

\begin{tabular}{|c|c|c|c|c|c|c|}
\hline Crosses & $\begin{array}{l}\text { Days to } 50 \% \\
\text { tasselling }\end{array}$ & $\begin{array}{l}\text { Days to } 50 \% \\
\text { silking }\end{array}$ & $\begin{array}{l}\text { Plant height } \\
\text { (cm) }\end{array}$ & $\begin{array}{c}\text { Ear height } \\
\text { (cm) }\end{array}$ & Prolificacy & $\begin{array}{c}\text { Kernel } \\
\text { rows cob }^{-1}\end{array}$ \\
\hline KDM-500×SMC-7 & $1.417 * *$ & $1.729 * *$ & $-12.937 * *$ & $-3.039 * *$ & 0.021 & $-0.711 * *$ \\
\hline KDM-500×CML-451 & $1.292 * *$ & $0.729 *$ & $9.868^{* *}$ & $4.993 * *$ & 0.015 & 0.104 \\
\hline KDM-500×DMR-N6 & $-2.708 * *$ & $-2.458 * *$ & $3.069 * *$ & $-1.954 * * *$ & -0.035 & $0.606 * *$ \\
\hline KDM-347×SMC-7 & -0.500 & $0.729 *$ & $12.616^{* *}$ & $2.761 * *$ & 0.021 & $0.598 * *$ \\
\hline KDM-347×CML-451 & 0.375 & -0.521 & $-19.763 * *$ & $-6.782 * *$ & 0.015 & $0.513 * *$ \\
\hline KDM-347×DMR-N6 & 0.125 & -0.208 & $7.147 * *$ & $4.021 * *$ & -0.035 & $-1.110^{* *}$ \\
\hline
\end{tabular}




\begin{tabular}{|c|c|c|c|c|c|c|}
\hline KDM-440B ×SMC-7 & $-2.917 * *$ & $-2.604 * *$ & -0.132 & $-2.172 * *$ & -0.046 & $-1.688^{* *}$ \\
\hline KDM-440B $\times$ CML-451 & 0.208 & 0.146 & $-6.227 * *$ & -0.766 & -0.052 & $0.405 * *$ \\
\hline KDM-440B×DMR-N6 & $2.708^{* * *}$ & $2.458 * *$ & $6.359 * *$ & $2.938^{* *}$ & $0.098^{*}$ & $1.282 * *$ \\
\hline KDM-445A×SMC-7 & -0.417 & $-1.271 * *$ & $-2.212 *$ & 0.895 & -0.929 & $-1.148 * *$ \\
\hline KDM-445A $\times$ CML-451 & $1.208^{* * *}$ & $1.729 * *$ & $4.376^{* *}$ & $-1.824 *$ & -0.035 & $-0.407 * *$ \\
\hline KDM-445A×DMR-N6 & $-0.792 *$ & -0.458 & $-2.164 *$ & 0.029 & $-0.065^{*}$ & $1.555 * *$ \\
\hline S.E. $\left(\mathbf{S}_{\mathrm{ij}}\right)$ & 0.315 & 0.362 & 1.075 & 0.718 & 0.028 & 0.092 \\
\hline High sca crosses & 9 & 8 & 9 & 10 & 4 & 12 \\
\hline
\end{tabular}

\begin{tabular}{|c|c|c|c|c|c|c|}
\hline Crosses & Kernels row $^{-1}$ & $\begin{array}{c}\text { Grain depth } \\
(\mathbf{c m})\end{array}$ & $\begin{array}{l}100 \text { grain } \\
\text { weight }(\mathrm{g})\end{array}$ & $\begin{array}{c}\text { Shelling } \\
\text { percentage }(\%)\end{array}$ & $\begin{array}{c}\text { Grain yield } \\
\text { plant }^{-1}(\mathrm{~g})\end{array}$ & $\begin{array}{c}\text { Protein } \\
\text { content }(\%)\end{array}$ \\
\hline V-351 $\times$ SMC-7 & $-3.837 * *$ & -0.003 & $-1.697 * *$ & $-2.784 * *$ & $-10.806 * *$ & $-0.254 * *$ \\
\hline V-351×CML-451 & $2.057 * *$ & $-0.024 * *$ & $-1.219 * *$ & $-1.942 * *$ & $-16.459 * *$ & 0.027 \\
\hline V-351×DMR-N6 & $1.780 * *$ & $0.024 * *$ & $2.916^{* *}$ & $4.726^{* *}$ & $27.265^{* *}$ & $0.227 * *$ \\
\hline V-335 $\times$ SMC-7 & $-3.179 * *$ & $0.032 * *$ & $2.928 * *$ & $1.561 * *$ & $3.667 * *$ & $-0.383 * *$ \\
\hline V-335×CML-451 & $3.665^{* *}$ & $0.013 *$ & $-0.669 *$ & $0.733 * *$ & $13.359 * *$ & $0.591 * *$ \\
\hline V-335×KDM343A & -0.487 & $-0.046 * *$ & $-2.259 * *$ & $-2.294 * *$ & $-17.027 * *$ & $-0.209 * *$ \\
\hline V-370 $\times$ SMC-7 & $-1.152 * *$ & 0.006 & $-3.392 * *$ & $1.124 * *$ & $-18.396 * *$ & $0.717 * *$ \\
\hline KDM2111×CML-451 & $2.702 * *$ & 0.005 & $3.416^{* *}$ & $-1.222 * *$ & $26.991 * *$ & $-0.709 * *$ \\
\hline V-370×DMR-N6 & $-1.550 * *$ & $-0.012 *$ & -0.024 & 0.099 & $-8.595 * *$ & -0.009 \\
\hline CM-502× SMC-7 & $-5.495 * *$ & $-0.021 * *$ & $2.270^{* *}$ & $2.993^{* *}$ & $-22.165^{* *}$ & -0.124 \\
\hline KDM2113 $\times$ CML-451 & 0.499 & $-0.063 * *$ & $-3.002 * *$ & $-1.443 * *$ & $11.437 * *$ & $-0.200 * *$ \\
\hline CM-502×DMR-N6 & $4.997 * *$ & $0.083 * *$ & $0.932 * *$ & $-1.550 * *$ & $10.728 * *$ & $0.325^{* *}$ \\
\hline
\end{tabular}

\begin{tabular}{|c|c|c|c|c|c|c|}
\hline Crosses & $\underset{1}{\text { Kernels row }}$ & $\begin{array}{c}\text { Grain depth } \\
(\mathbf{c m})\end{array}$ & $\begin{array}{l}100 \text { grain } \\
\text { weight }(\mathrm{g})\end{array}$ & $\begin{array}{c}\text { Shelling } \\
\text { percentage }(\%)\end{array}$ & $\begin{array}{c}\text { Grain yield } \\
\text { plant }\end{array}$ & $\begin{array}{c}\text { Protein } \\
\text { content }(\%)\end{array}$ \\
\hline KDM-500×SMC-7 & $2.513^{* *}$ & $-0.050 * *$ & -0.105 & -0.092 & -0.180 & $0.251 * *$ \\
\hline KDM-500×CML-451 & $1.882 * *$ & $-0.010 *$ & -0.177 & $-0.996 * *$ & $14.429 * *$ & 0.075 \\
\hline KDM-500×DMR-N6 & $-4.395 * *$ & $0.061 * *$ & 0.282 & $1.088^{* * *}$ & $-14.249 * *$ & $-0.325 * *$ \\
\hline KDM-347×SMC-7 & $9.440 * *$ & 0.130 ** & $3.888^{* * *}$ & $0.780^{* * *}$ & $72.542 * *$ & $-0.141^{*}$ \\
\hline KDM-347×CML-451 & $-6.941 * *$ & $-0.095 * *$ & $0.965^{* *}$ & $4.431 * *$ & $-24.828 * *$ & $0.333 * *$ \\
\hline KDM-347×DMR-N6 & $-2.500 * *$ & -0.003 & $-4.853 * *$ & $-5.211 * *$ & $-47.714 * *$ & $-0.192 * *$ \\
\hline KDM-440B $\times$ SMC-7 & $3.913^{* *}$ & $-0.064 * *$ & $-4.272 * *$ & $-3.168 * *$ & $-4.549 * *$ & $-0.249 * *$ \\
\hline KDM-440B $\times$ CML-451 & $-2.031 * *$ & $0.110 * *$ & $2.206 * *$ & $1.873 * *$ & $-5.086^{* *}$ & 0.025 \\
\hline KDM-440B×DMR-N6 & $-1.882 * *$ & $-0.044 * *$ & $2.066^{* *}$ & $1.294^{* * *}$ & $9.635^{* * *}$ & $0.225 * *$ \\
\hline KDM-445A×SMC-7 & $-2.203 * *$ & $-0.026 * *$ & 0.378 & -0.414 & $-20.114 * *$ & $0.184 * *$ \\
\hline KDM-445A×CML-451 & $-1.834 * *$ & $0.082 * *$ & $-1.519 * *$ & $-1.433 * *$ & $-19.844 * *$ & $-0.142 *$ \\
\hline KDM-445A×DMR-N6 & $4.037 * *$ & $-0.057 * *$ & $1.141 * *$ & $1.848 * *$ & $39.957 * *$ & -0.042 \\
\hline S.E. $\left(\mathbf{S}_{\mathrm{ij}}\right)$ & 0.261 & 0.005 & 0.249 & 0.247 & 1.291 & 0.066 \\
\hline High sca crosses & 10 & 8 & 10 & 11 & 10 & 8 \\
\hline
\end{tabular}

*, ** Significant at 5 and 1 percent levels, respectively

\section{Specific combining ability effects}

The estimates of specific combining ability effects of the twenty-four crosses for various traits, given in table 3 , revealed that none of the cross combination possessed high SCA effects for all the traits. However, crosses which exhibited highly significant and desirable SCA effects included KDM-440B $\times$ SMC-7, KDM$500 \times$ DMR-N6 and V-351 $\times$ DMR-N6 for days 
to 50 per cent tasseling; V-351 $\times$ DMRN6,KDM-440B $\times$ SMC-7 and KDM-500 $\times$ DMR-N6 for days to 50 per cent silking; V-335 $\times$ DMR-N6, V-351 $\times$ SMC-7 and KDM-347 $\times$ CML-451 for plant height; V-351 $\times$ SMC-7, V$335 \times$ DMR-N6 and V-370 $\times$ CML-451 for ear height; KDM-440B $\times$ DMR-N6 and V-370 $\times$ DMR-N6 for prolificacy; V-335 $\times$ SMC-7, KDM-445A $\times$ DMR-N6 and CM-502 $\times$ CML451 for kernel rows cob ${ }^{-1}$; KDM-347 $\times$ SMC-7, CM-502 $\times$ DMR-N6 and KDM-445A $\times$ DMRN6 for kernels row $^{-1}$; KDM-347 $\times$ SMC-7, KDM-440B $\times$ CML-451 and CM-502 $\times$ DMRN6 for grain depth; KDM-347 $\times$ SMC-7, V-370 $\times$ CML-451 and V-335 $\times$ SMC-7 for 100 grain weight; V-351 $\times$ DMR-N6, KDM-347 $\times$ CML451 and CM-502 $\times$ SMC-7 for shelling percentage; KDM-347 × SMC-7, KDM-445A $\times$ DMR-N6 and V-351 $\times$ DMR-N6 for grain yield plant $^{-1}$ and V-370 $\times$ SMC-7, V-335 $\times$ CML-451 and KDM-347 $\times$ CML-451 for protein content. While assessing the performance of parents on the basis general combining ability, it was observed that most of the specific cross combinations were the result of crosses between low $\times$ high or low $\times$ low or low $\times$ medium or high $\times$ high or high $\times$ medium general combiners. Among these crosses, KDM-347 $\times$ SMC-7 which showed the highest positive SCA effect for yield had high $\times$ medium combiners; KDM-445A $\times$ DMR-N6 had high $\times$ high combiners; V-351 $\times$ DMR-N6 had low $\times$ high combiners suggesting that involvement of one good general combiner appears to be essential to get the better specific combination. The results are in general agreement with the findings of several workers (Dass et al., 1997; Aguiar et al., 2003; Asif et al., 2007; Ali et al., 2007 and Dar et al., 2017). Labana et al., (1978) have suggested that manifestation of high SCA effects in some cross combinations results due to accumulation and interaction of favorable alleles from parents that are high or medium general combiners. Thus the superiority of crosses involving high $\times$ high and high $\times$ medium combiners as parents might have possibly resulted from the concentration and interaction of favourable alleles contributed by parents. The case of high SCA between high $\mathrm{x}$ low combiners could produce good segregants only if the additive genetic effects are present in the good general combiners and complementary epistatic effects in the poor combiners and they act in the same direction to maximize desirable plant attributes (Singh and Chaudhary, 1992). The high yield of such crosses would be nonfixable and thus could be exploited through heterosis breeding. The superior cross combination involving low $\times$ low combiners could result from over-dominance and/or epitasis (Rehman et al., 1981).

It can be concluded that the highly significant differences were observed among line and line $x$ testers for all the traits which indicate the possibility of selection for improvement of yield and yield related traits. Among parents, KDM445A, CM-502 and KDM-347 showed highly desirable GCA effects for grain yield plant ${ }^{-1}$ and can be selected for the development of hybrids. Moreover, crosses KDM-347 × SMC-7, KDM$445 \mathrm{~A} \times$ DMR-N6 and V-351 $\times$ DMR-N6 reflected high SCA effects for grain yield plant ${ }^{-1}$. Thus these crosses can be used directly or exploited for future hybrid breeding programmes to achieve quantum jump in maize improvement.

\section{References}

Abuali, A. I., Abdelmulla, A. A., Khalafalla, M. M., Idris, A. E.and Osman, A. M. 2012. Combining ability and heterosis for yield and yield components in maize (Zea mays L.). Australian Journal of Basic and Applied Sciences 6(10): 36-41.

Aguiar, A.M., Carlini-Garcia, L. A., Silva, A. R. Santos, M.F., Garcia, A. A. F. and Souja, J.R.. 2003. Combining ability of inbred lines of maize and stability of their respective single-crosses. Scientia Agricricola 60: 83-89.

Ali, G., Rather, A.G., Ishfaq, A., Dar, S.A., Wani, S.A. and Khan, M.N. 2007. Gene action for grain yield and its attributes in maize ( $\mathrm{Zea}$ mays L.). International Journal of Agricultural Science 3 (2): 278-281.

Anonymous, 2015. Food and Agricultural 
Organization year book of the United Nations 2015 Rome, Italy. http://faostat.fao.org/faostat/servlet/xteServl et 3

Asif, M.I., Nehvi, F.A., Wani, S.A., Qadir, R. and Dar, Z.A. 2007. Combining ability for grain yield and yield related traits in maize. (Zea mays L.). International Journal of Agricultural Sciences 27: 101-105.

Dar,Z.A., Lone,A.A., Elahi,B.A., Dar,S.A., Ali,G., Abidi,I., Lone,R.A., Gulzar,S. and Yousuf,N. 2017. Genetic variation in maize inbred lines under temperate conditions. Journal of Experimental Biology and Agriculture Sciences. 5(2): 215-219

Dass S., Ahuja, V.P. and Singh, M. 1997. Combining ability for yield in maize. Indian Journal of Genetics and Plant Breeding 57(1): 98-100.

Haddadi, M.H., Eesmaeilov, M. and Choukan, R. 2013. Determination of genetic heritability for some agronomic traits in corn by diallel analysis. International Journal of Agriculture and Crop Sciences 5(15): 16871693.

Kanagarasu, S., Nallathambi, G. and Ganesan, K.N. 2010. Combining ability analysis for yield and its component traits in maize (Zea mays L.) Electronic Journal of Plant Breeding 1(4): 915-920.

Kempthorne, O. 1957. An Introduction to Genetic Statistics. John Wiley and Sons, Inc. New York, USA, pp. 468-473.

Krupakar, A., Kumar, B. and Marker, S. 2014. Combining ability for yield and quality traits in single cross hybrids of maize (Zea mays L.). The Bioscan 8 (4): 1347-1355.

Labana, K.S., Ram, T., Badwal, S.S. and Mehan, D.K. 1978. Combining ability analysis in the Indian mustard. Crop Improvement 5:
137-144.

Iqbal, A.M., Nehvi, F.A., Wani, S.A., Qadir, R. and Dar, Z.A. 2007. Combining ability analysis for yield and yield related traits in maize (Zea mays L.). International Journal of Plant Breeding and Genetics 1: 101-105.

Premlatha, M., Kalamani, A. and Nirmalakumari, A. 2011. Heterosis and combining ability for grain yield and quality in maize (Zea mays L.). Advances in Environmental Biology 5(6): 1264-1266

Pavan, R., Lohithaswa, H.C., Prakash, G., Wali, M.C. and Shekara, B.G. 2011. Combining ability analysis of newer inbred lines derived from national yellow pool for grain yield and other quantitative traits in maize (Zea mays L.). Electronic Journal of Plant Breeding 2(3): 310-319.

Rehman, M., Patway, A.K. and Miah, A.J. 1981. Combining ability in rice. Indian Journal of Agricultural Science 15: 543-546.

Shanthi, P., Babu, G.S., Satyanarayana, E. and Kumar, R.S. 2010. Combining ability and stability studies for grain yield and quality parameters in QPM (Zea mays L.) inbred line crosses. Indian Journal of Genetics 70(1): 22-29.

Shashidhara, C.K. 2008. Early generation testing for combining ability in maize (Zea mays L.). M.Sc. thesis submitted to University of Agriculture Sciences, Dharwad (Institute).

Singh, P. K., Singh, A.K., Shahi, J.P. and Ranjan, R. 2012. Combining ability and heterosis in quality protein maize. The bioscan 7(2): 337-340.

Singh, S. and Chaudhary, B.S. 1992. Combining ability for some metric traits in rice. Madras Agricultural Journal, 82. 165-169.

\section{How to cite this article:}

Dar, Z.A., A.A. Lone, N.S. Khuroo, G. Ali, I. Abidi, M.A. Ahangar, M.A. Wani, A.B. Yasin, A. Gazal, R.A. Lone, N. Yousuf and Gulzar, S. 2017. Line x Tester Analysis in Maize (Zea mays L.) for Various Morpho-Agronomic Traits under Temperate Conditions. Int.J.Curr.Microbiol.App.Sci. 6(7): 1430-1437. doi: https://doi.org/10.20546/ijcmas.2017.607.171 